\title{
Synthesis and Reactions of Group 4 Imido Complexes Supported by Cyclooctatetraene Ligands
}

\author{
Andrew R. Cowley, Simon C. Dunn, Jennifer C. Green,* Nilay Hazari and Philip \\ Mountford*
}

Inorganic Chemistry Laboratory, University of Oxford, South Parks Road, Oxford OX1 $3 Q R$ U.K.

\section{SUPPORTING INFORMATION}

\section{Reaction between $\left[\mathrm{Ti}\left(\mathrm{N}^{\mathrm{t}} \mathrm{Bu}\right)(\mathrm{COT})\right](1)$ and ${ }^{\mathrm{t}} \mathrm{BuNCO}$}

A solution of ${ }^{\mathrm{t}} \mathrm{BuNCO}(44 \mathrm{mg}, 0.45 \mathrm{mmol})$ in THF $(10 \mathrm{~mL})$ was added to a stirred solution of [ $\left.\mathrm{Ti}\left(\mathrm{N}^{\mathrm{t}} \mathrm{Bu}\right)(\mathrm{COT})\right](\mathbf{1})(0.10 \mathrm{~g}, 0.45 \mathrm{mmol})$ in THF $(10 \mathrm{~mL})$. Upon addition of the ${ }^{t} \mathrm{BuNCO}$ solution the color of the reaction mixture immediately changed from yellow to brown. The reaction mixture was stirred for 5 hours and the volatiles removed under reduced pressure. The light brown residue was washed with pentane $(2 \times 15 \mathrm{~mL})$ and the solid was dried in vacuo. The solid was characterized as $[\mathrm{Ti}(\mathrm{O})(\mathrm{COT})]_{\mathrm{n}}(\mathbf{4})$. The byproduct of the reaction, ${ }^{t} \mathrm{BuNCN} \mathrm{N}^{\mathrm{t}} \mathrm{Bu}$, was characterized by ${ }^{1} \mathrm{H}$ NMR spectroscopy when the reaction was performed on an NMR tube scale. The presence of ${ }^{t} \mathrm{BuNCN}{ }^{t} \mathrm{Bu}$ in the NMR tube was confirmed by adding an authentic sample of ${ }^{t} \mathrm{BuNCN}^{t} \mathrm{Bu}$ to the reaction mixture.

\section{Reaction between $\left[\mathrm{Ti}\left(\mathrm{N}^{t} \mathrm{Bu}\right)(\mathrm{COT})\right](1)$ and ArNCO}

A solution of ArNCO $(91 \mathrm{mg}, 0.45 \mathrm{mmol})$ in THF $(10 \mathrm{~mL})$ was added to a stirred solution of [Ti( $\left.\left.\mathrm{N}^{t} \mathrm{Bu}\right)(\mathrm{COT})\right](\mathbf{1})(0.10 \mathrm{~g}, 0.45 \mathrm{mmol})$ in THF $(10 \mathrm{~mL})$. Upon addition of the ArNCO solution the color of the reaction mixture immediately changed from yellow to brown. The reaction mixture was stirred for 5 hours and the volatiles removed under reduced pressure. The light brown residue was washed with pentane $(2 \times 15 \mathrm{~mL})$ and the solid was dried in vacuo. The solid was characterized as $[\operatorname{Ti}(\mathrm{O})(\mathrm{COT})]_{\mathrm{n}}(\mathbf{4})$. The byproduct of the reaction, ${ }^{t} \mathrm{BuNCNAr}$, was characterized by ${ }^{1} \mathrm{H}$ NMR spectroscopy 
(compared to the literature species) ${ }^{1}$ when the reaction was performed on an NMR tube scale.

\section{Reaction between $\left[\mathrm{Ti}\left(\mathrm{N}^{\mathrm{t}} \mathrm{Bu}\right)(\mathrm{COT})\right](1)$ and nitrosobenzene}

A solution of nitrosobenzene $(48 \mathrm{mg}, 0.45 \mathrm{mmol})$ in benzene $(10 \mathrm{~mL})$ was added to a stirred solution of $\left[\mathrm{Ti}\left(\mathrm{N}^{\mathrm{t}} \mathrm{Bu}\right)(\mathrm{COT})\right](\mathbf{1})(0.10 \mathrm{~g}, 0.45 \mathrm{mmol})$ in benzene $(15 \mathrm{~mL})$. Upon addition of the nitrosobenzene solution the reaction mixture immediately changed color from yellow to brown. The reaction mixture was stirred for 6 hours during which time a brown solid precipitated out of solution. The volatiles were removed under reduced pressure and the residue washed with pentane $(2 \times 15 \mathrm{~mL})$. The solid was dried in vacuo and characterized as $[\mathrm{Ti}(\mathrm{O})(\mathrm{COT})]_{\mathrm{n}}(\mathbf{4})$. The by-product of the reaction, ${ }^{2}{ }^{\mathrm{t}}{ }^{\mathrm{B}} \mathrm{BuN}=\mathrm{NPh}$, was characterized by ${ }^{1} \mathrm{H}$ NMR spectroscopy (compared to the literature species) ${ }^{2}$ when the reaction was performed on an NMR tube scale.

\section{Reaction between [Ti(NAr)(COT)] (3) and ${ }^{\mathrm{t}} \mathrm{BuNCO}$}

A solution of ${ }^{\mathrm{t}} \mathrm{BuNCO}(30 \mathrm{mg}, 0.31 \mathrm{mmol})$ in $\mathrm{THF}(10 \mathrm{~mL})$ was added to a stirred solution of [Ti(NAr)(COT)] (3) $(0.10 \mathrm{~g}, 0.31 \mathrm{mmol})$ in THF $(10 \mathrm{~mL})$. Upon addition of the ${ }^{t} \mathrm{BuNCO}$ solution the color of the reaction mixture immediately changed from orange to brown. The reaction mixture was stirred for 5 hours and the volatiles removed under reduced pressure. The light brown residue was washed with pentane $(2 \times 15 \mathrm{~mL})$ and the solid was dried in vacuo. The solid was characterized as $[\operatorname{Ti}(\mathrm{O})(\mathrm{COT})]_{\mathrm{n}}(4)$. The byproduct of the reaction, ${ }^{\mathrm{t}} \mathrm{BuNCNAr}$, was characterized by ${ }^{1} \mathrm{H}$ NMR spectroscopy (compared to the literature species) ${ }^{1}$ when the reaction was performed on an NMR tube scale.

\section{Reaction between $\left[\mathrm{Ti}\left(\mathrm{N}^{\mathrm{t}} \mathrm{Bu}\right)\left(\mathrm{COT}^{\prime \prime}\right)\right](2)$ and ${ }^{\mathrm{t}} \mathrm{BuNCO}$}

A solution of ${ }^{\mathrm{t}} \mathrm{BuNCO}(31 \mathrm{mg}, 0.27 \mathrm{mmol})$ in THF $(10 \mathrm{~mL})$ was added to a stirred solution of $\left[\mathrm{Ti}\left(\mathrm{N}^{\mathrm{t}} \mathrm{Bu}\right)\left(\mathrm{COT}^{\prime \prime}\right)\right](2)(0.10 \mathrm{~g}, 0.27 \mathrm{mmol})$ in THF $(10 \mathrm{~mL})$. Upon addition of the ${ }^{t} \mathrm{BuNCO}$ solution the color of the reaction mixture immediately changed from yellow to brown. The reaction mixture was stirred for 5 hours and the volatiles removed under reduced pressure. The light brown residue was washed with pentane $(2 \times 15 \mathrm{~mL})$ and the solid was dried in vacuo. The solid was characterized as $\left[\mathrm{Ti}(\mathrm{O})\left(\mathrm{COT}^{\prime \prime}\right)\right]_{\mathrm{n}}(\mathbf{6})$. The by-

product of the reaction, ${ }^{\mathrm{t}} \mathrm{BuNCN}{ }^{\mathrm{t}} \mathrm{Bu}$, was characterized by ${ }^{1} \mathrm{H}$ NMR spectroscopy when 
the reaction was performed on an NMR tube scale. The presence of ${ }^{t} \mathrm{BuNCN}^{t} \mathrm{Bu}$ in the NMR tube was confirmed by adding an authentic sample of ${ }^{t} \mathrm{BuNCN}{ }^{t} \mathrm{Bu}$ to the reaction mixture.

\section{Reaction between $\left[\mathrm{Ti}\left(\mathrm{N}^{\mathrm{t}} \mathrm{Bu}\right)(\mathrm{COT})\right](1)$ and ${ }^{\mathrm{t}} \mathrm{BuNCS}$}

A solution of ${ }^{\mathrm{t}} \mathrm{BuNCS}(52 \mathrm{mg}, 0.45 \mathrm{mmol})$ in THF $(10 \mathrm{~mL})$ was added to a stirred solution of $\left[\mathrm{Ti}\left(\mathrm{N}^{\mathrm{t}} \mathrm{Bu}\right)(\mathrm{COT})\right](\mathbf{1})(0.10 \mathrm{~g}, 0.45 \mathrm{mmol})$ in THF $(5 \mathrm{~mL})$. Upon addition of the ${ }^{t} \mathrm{BuNCS}$ solution the color of the reaction mixture immediately changed from yellow to brown. The reaction mixture was stirred for 3 hours and the volatiles removed under reduced pressure. The light brown residue was washed with pentane $(2 \times 15 \mathrm{~mL})$ and the solid was dried in vacuo. The solid was characterized as $[\mathrm{Ti}(\mathrm{S})(\mathrm{COT})]_{\mathrm{n}}(\mathbf{8})$. The byproduct of the reaction, ${ }^{\mathrm{t}} \mathrm{BuNCN}{ }^{\mathrm{t}} \mathrm{Bu}$, was characterized by ${ }^{1} \mathrm{H}$ NMR spectroscopy when the reaction was performed on an NMR tube scale. The presence of ${ }^{t} \mathrm{BuNCN}^{\mathrm{t}} \mathrm{Bu}$ in the NMR tube was confirmed by adding an authentic sample of ${ }^{t} \mathrm{BuNCN}{ }^{t} \mathrm{Bu}$ to the reaction mixture.

\section{Reaction between [Ti(NAr)(COT)] (3) and ${ }^{\mathrm{t}}$ BuNCS}

A solution of ${ }^{\mathrm{t}} \mathrm{BuNCS}(52 \mathrm{mg}, 0.45 \mathrm{mmol})$ in THF $(5 \mathrm{~mL})$ was added to a stirred solution of [Ti(NAr)(COT)] (3) $(0.10 \mathrm{~g}, 0.31 \mathrm{mmol})$ in THF $(5 \mathrm{~mL})$. Upon addition of the ${ }^{\mathrm{t}} \mathrm{BuNCS}$ solution the color of the reaction mixture immediately changed from orange to brown. The reaction mixture was stirred for 3 hours and the volatiles removed under reduced pressure. The light brown residue was washed with pentane $(2 \times 15 \mathrm{~mL})$ and the solid was dried in vacuo. The solid was characterized as $[\mathrm{Ti}(\mathrm{S})(\mathrm{COT})]_{\mathrm{n}}(\mathbf{8})$. The by-product of the reaction, ${ }^{\mathrm{t}} \mathrm{BuNCN}-2,6-{ }^{\mathrm{i}} \mathrm{Pr}_{2} \mathrm{C}_{6} \mathrm{H}_{3}$, was characterized by ${ }^{1} \mathrm{H}$ NMR spectroscopy (compared to the literature species) ${ }^{1}$ when the reaction was performed on an NMR tube scale.

NMR tube scale reactions between $\left[\mathrm{Ti}\left(\mathrm{N}^{\mathrm{t}} \mathrm{Bu}\right)(\mathrm{COT})\right](1),\left[\mathrm{Ti}\left(\mathrm{N}^{t} \mathrm{Bu}\right)\left(\mathrm{COT}^{\prime \prime}\right)\right](2)$ and [Ti(NAr)(COT)] (3) and ${ }^{\mathrm{t}} \mathrm{BuOH}$

The compounds $\left[\mathrm{Ti}\left(\mathrm{N}^{t} \mathrm{Bu}\right)(\mathrm{COT})\right] \quad(\mathbf{1}), \quad\left[\mathrm{Ti}\left(\mathrm{N}^{\mathrm{t} B u}\right)\left(\mathrm{COT}^{\prime \prime}\right)\right] \quad(\mathbf{2})$ and $[\mathrm{Ti}(\mathrm{N}-2,6-$ $\left.\left.{ }^{\mathrm{i}} \mathrm{Pr}_{2} \mathrm{C}_{6} \mathrm{H}_{3}\right)(\mathrm{COT})\right](3)$ were all reacted with both one and two equivalents of ${ }^{\mathrm{t}} \mathrm{BuOH}$ on the NMR tube scale. A representative procedure is outlined below. 
A solution of $\left[\mathrm{Ti}\left(\mathrm{N}^{t} \mathrm{Bu}\right)(\mathrm{COT})\right](\mathbf{1})(10 \mathrm{mg}, 0.045 \mathrm{mmol})$ in $\mathrm{C}_{6} \mathrm{D}_{6}(0.4 \mathrm{~mL})$ was treated with one equivalent of ${ }^{\mathrm{t}} \mathrm{BuOH}(3.3 \mathrm{mg}, 0.045 \mathrm{mmol})$. Upon addition of ${ }^{\mathrm{t}} \mathrm{BuOH}$ the reaction mixture darkened and over 18 hours gradually turned brown. The reaction mixture was monitored by ${ }^{1} \mathrm{H}$ and ${ }^{13} \mathrm{C}$ NMR spectroscopy for 2 days and after 2 days there were no resonances corresponding to either ${ }^{t} \mathrm{BuOH}$ or $\mathbf{1}$ present in either the ${ }^{1} \mathrm{H}$ or ${ }^{13} \mathrm{C}$ NMR spectra.

\section{NMR tube scale reaction between $\left[\mathrm{Ti}\left(\mathrm{N}^{t} \mathrm{Bu}\right)(\mathrm{COT})\right](1)$ and $\mathrm{HS}-2,4,6-{ }^{\mathrm{i}} \mathrm{Pr}_{3} \mathrm{C}_{6} \mathrm{H}_{2}$}

A solution of $\left[\mathrm{Ti}\left(\mathrm{N}^{t} \mathrm{Bu}\right)(\mathrm{COT})\right](\mathbf{1})(10 \mathrm{mg}, 0.045 \mathrm{mmol})$ in $\mathrm{C}_{6} \mathrm{D}_{6}(0.4 \mathrm{~mL})$ was treated with 2 equivalents of $\mathrm{HS}-2,4,6{ }^{\mathrm{i}} \mathrm{Pr}_{3} \mathrm{C}_{6} \mathrm{H}_{2}(21 \mathrm{mg}, 0.090 \mathrm{mmol})$. Upon addition of HS2,4,6- ${ }^{\mathrm{i}} \mathrm{Pr}_{3} \mathrm{C}_{6} \mathrm{H}_{2}$ the reaction mixture changed color from yellow to brown. The reaction mixture was monitored by ${ }^{1} \mathrm{H}$ NMR spectroscopy for 2 days and a brown solid precipitated out of solution. The presence of $2,4,6-{ }^{\mathrm{i}} \mathrm{Pr}_{3} \mathrm{C}_{6} \mathrm{H}_{2} \mathrm{~S}-\mathrm{S}-2,4,6-{ }^{\mathrm{i}} \mathrm{Pr}_{3} \mathrm{C}_{6} \mathrm{H}_{2}$ in the NMR tube was confirmed by adding an authentic sample of $2,4,6-{ }^{\mathrm{i}} \mathrm{Pr}_{3} \mathrm{C}_{6} \mathrm{H}_{2} \mathrm{~S}-\mathrm{S}-2,4,6-$ ${ }^{\mathrm{i}} \mathrm{Pr}_{3} \mathrm{C}_{6} \mathrm{H}_{2}$ (provided by Dr. John Woollard-Shore) to the reaction mixture.

\section{NMR tube scale reaction between [Ti(NAr)(COT)] (3) and ${ }^{\mathrm{t} B u S H}$}

A solution of [Ti(NAr)(COT)] (3) $(10 \mathrm{mg}, 0.031 \mathrm{mmol})$ in $\mathrm{C}_{6} \mathrm{D}_{6}(0.4 \mathrm{~mL})$ was treated with 2 equivalents of ${ }^{\mathrm{t}} \mathrm{BuSH}(5.5 \mathrm{mg}, 0.062 \mathrm{mmol})$. Upon addition of ${ }^{\mathrm{t}} \mathrm{BuSH}$ the reaction mixture changed color from orange to brown. The reaction mixture was monitored by ${ }^{1} \mathrm{H}$ NMR spectroscopy for 2 days and a brown solid precipitated out of solution. The presence of ${ }^{\mathrm{t}} \mathrm{BuS}-\mathrm{S}^{\mathrm{t}} \mathrm{Bu}$ in the NMR tube was confirmed by adding an authentic sample of ${ }^{t} \mathrm{BuS}-\mathrm{S}^{t} \mathrm{Bu}$ to the reaction mixture.

\section{NMR tube scale reaction between [Ti(NAr)(COT)] (3) and $\mathrm{HS}-2,4,6-{ }^{\mathrm{i}} \mathrm{Pr}_{3} \mathrm{C}_{6} \mathrm{H}_{2}$}

A solution of [Ti(NAr)(COT)] (3) $(10 \mathrm{mg}, 0.031 \mathrm{mmol})$ in $\mathrm{C}_{6} \mathrm{D}_{6}(0.4 \mathrm{~mL})$ was treated with 2 equivalents of HS-2,4,6 ${ }^{\mathrm{i}} \mathrm{Pr}_{3} \mathrm{C}_{6} \mathrm{H}_{2}$ (14 mg, $\left.0.061 \mathrm{mmol}\right)$. Upon addition of HS2,4,6- ${ }^{\mathrm{i}} \mathrm{Pr}_{3} \mathrm{C}_{6} \mathrm{H}_{2}$ the reaction mixture changed color from orange to brown. The reaction mixture was monitored by ${ }^{1} \mathrm{H}$ NMR spectroscopy for 2 days and a brown solid precipitated out of solution. The presence of $2,4,6-{ }^{\mathrm{i}} \mathrm{Pr}_{3} \mathrm{C}_{6} \mathrm{H}_{2} \mathrm{~S}-\mathrm{S}-2,4,6-{ }^{\mathrm{i}} \mathrm{Pr}_{3} \mathrm{C}_{6} \mathrm{H}_{2}$ in the NMR tube was confirmed by adding an authentic sample of $2,4,6-{ }^{\mathrm{i}} \mathrm{Pr}_{3} \mathrm{C}_{6} \mathrm{H}_{2} \mathrm{~S}-\mathrm{S}-2,4,6-$ ${ }^{\mathrm{i}} \mathrm{Pr}_{3} \mathrm{C}_{6} \mathrm{H}_{2}$ (provided by Dr. John Woollard-Shore) to the reaction mixture.

\section{Reaction between $\left[\mathrm{Ti}\left(\mathrm{N}^{t} \mathrm{Bu}\right)(\mathrm{COT})\right](1)$ and MeNC}


A solution of MeNC (37 mg, $0.90 \mathrm{mmol})$ in THF $(10 \mathrm{~mL})$ was added to a stirred solution of $\left[\mathrm{Ti}\left(\mathrm{N}^{\mathrm{t}} \mathrm{Bu}\right)(\mathrm{COT})\right](\mathbf{1})(0.20 \mathrm{~g}, 0.90 \mathrm{mmol})$ in THF $(10 \mathrm{~mL})$. Upon addition of the MeNC solution the color of the reaction mixture immediately changed from yellow to brown. The reaction mixture was stirred for 2 hours and the volatiles removed under reduced pressure. The light brown residue was extracted with ether $(15 \mathrm{~mL})$ and filtered. The solvent was again removed under reduced pressure and the brown powder washed with pentane (2 x $15 \mathrm{~mL})$ and dried in vacuo. Analysis by ${ }^{1} \mathrm{H}$ and ${ }^{13} \mathrm{C}$ NMR spectroscopy indicated that the powder was a mixture of several different COT containing complexes.

\section{Reaction between $\left[\mathrm{Ti}\left(\mathrm{N}^{t} \mathrm{Bu}\right)(\mathrm{COT})\right](1)$ and $\mathrm{Ar} \mathbf{r}^{\mathrm{NC}}$}

A solution of $\mathrm{Ar}^{\prime} \mathrm{NC}_{6}(0.12 \mathrm{~g}, 0.90 \mathrm{mmol})$ in $\mathrm{THF}(10 \mathrm{~mL})$ was added to a stirred solution of $\left[\mathrm{Ti}\left(\mathrm{N}^{t} \mathrm{Bu}\right)(\mathrm{COT})\right](\mathbf{1})(0.20 \mathrm{~g}, 0.90 \mathrm{mmol})$ in THF $(10 \mathrm{~mL})$. Upon addition of the Ar'NC solution the color of the reaction mixture immediately changed from yellow to brown. The reaction mixture was stirred for 2 hours and the volatiles removed under reduced pressure. The light brown residue was extracted with ether $(15 \mathrm{~mL})$ and filtered. The solvent was again removed under reduced pressure and the brown powder washed with pentane (2 x $15 \mathrm{~mL})$ and dried in vacuo. Analysis by ${ }^{1} \mathrm{H}$ and ${ }^{13} \mathrm{C}$ NMR spectroscopy indicated that the powder was a mixture of several different COT containing complexes.

\section{Optimized Geometries}

[Ti(N $\left.\left.\mathbf{N}^{\mathrm{B} u}\right)(\mathrm{COT})\right](\mathbf{1})$

$\begin{array}{llll}\mathrm{Ti} & -0.018130 & 0.075016 & 1.390205 \\ \mathrm{~N} & -0.008605 & 0.143520 & -0.312555 \\ \mathrm{C} & -0.002296 & 0.213559 & -1.735410 \\ \mathrm{C} & -0.001100 & 1.672286 & -2.160859 \\ \mathrm{H} & 0.009499 & 1.759920 & -3.261768 \\ \mathrm{H} & 0.888744 & 2.181057 & -1.754958 \\ \mathrm{H} & -0.899675 & 2.179364 & -1.772544 \\ \mathrm{C} & -1.242555 & -0.479740 & -2.274203 \\ \mathrm{H} & -1.252577 & -1.535340 & -1.956651 \\ \mathrm{H} & -1.266644 & -0.439900 & -3.377680 \\ \mathrm{H} & -2.148183 & 0.010836 & -1.881005 \\ \mathrm{C} & 1.244649 & -0.477630 & -2.261310 \\ \mathrm{H} & 2.145632 & 0.016415 & -1.862054 \\ \mathrm{H} & 1.277967 & -0.441373 & -3.364634 \\ \mathrm{H} & 1.253943 & -1.531980 & -1.939486 \\ \mathrm{C} & -0.023098 & 1.873101 & 2.821806 \\ \mathrm{C} & 1.278134 & 1.332053 & 2.814057\end{array}$




$\begin{array}{llll}\mathrm{C} & 1.815729 & 0.029764 & 2.779988 \\ \mathrm{C} & 1.274830 & -1.271041 & 2.740435 \\ \mathrm{C} & -0.027821 & -1.808486 & 2.717479 \\ \mathrm{C} & -1.329145 & -1.267521 & 2.725726 \\ \mathrm{C} & -1.866445 & 0.034835 & 2.759631 \\ \mathrm{C} & -1.325565 & 1.335590 & 2.798836 \\ \mathrm{H} & -2.089284 & 2.106373 & 2.654682 \\ \mathrm{H} & -2.948505 & 0.041083 & 2.594250 \\ \mathrm{H} & -2.095576 & -2.027021 & 2.541472 \\ \mathrm{H} & -0.028335 & -2.886709 & 2.528793 \\ \mathrm{H} & 2.040905 & -2.033030 & 2.565302 \\ \mathrm{H} & 2.899391 & 0.032943 & 2.625682 \\ \mathrm{H} & 2.045577 & 2.100752 & 2.678800 \\ \mathrm{H} & -0.020996 & 2.959790 & 2.690635\end{array}$

\section{[Ti(N-2,6- $\left.\left.{ }^{\mathrm{i}} \mathrm{Pr}_{2} \mathbf{C}_{6} \mathrm{H}_{3}\right)(\mathrm{COT})\right]$ (3)}

$\begin{array}{llll}\mathrm{Ti} & 0.037085 & -0.000613 & 0.032381 \\ \mathrm{~N} & 0.066122 & 0.060871 & -1.679369 \\ \mathrm{C} & 0.088218 & 0.112916 & -3.042790 \\ \mathrm{C} & -0.444707 & 1.234048 & -3.719311 \\ \mathrm{C} & -0.409568 & 1.260872 & -5.104919 \\ \mathrm{C} & 0.133851 & 0.217638 & -5.836658 \\ \mathrm{C} & 0.653736 & -0.877265 & -5.167212 \\ \mathrm{C} & 0.643438 & -0.953884 & -3.782356 \\ \mathrm{C} & 1.218713 & -2.156477 & -3.101542 \\ \mathrm{C} & 0.442905 & -3.421556 & -3.499853 \\ \mathrm{H} & 0.040219 & -3.955360 & -2.624213 \\ \mathrm{H} & -0.409868 & -3.175755 & -4.153573 \\ \mathrm{H} & 1.072736 & -4.131505 & -4.058942 \\ \mathrm{C} & 2.718246 & -2.282551 & -3.412508 \\ \mathrm{H} & 3.070974 & -1.433032 & -4.020012 \\ \mathrm{H} & 3.331940 & -2.306881 & -2.497842 \\ \mathrm{H} & 2.944162 & -3.194558 & -3.987340 \\ \mathrm{H} & 1.093232 & -1.988677 & -2.015352 \\ \mathrm{H} & 1.087313 & -1.712069 & -5.735585 \\ \mathrm{H} & 0.152014 & 0.258119 & -6.928876 \\ \mathrm{H} & -0.825503 & 2.135529 & -5.624351 \\ \mathrm{C} & -1.041821 & 2.382767 & -2.960882 \\ \mathrm{C} & 1.659366 & 0.769134 & 1.473387 \\ \mathrm{C} & 1.757284 & -0.635039 & 1.423169 \\ \mathrm{C} & 0.832983 & -1.696487 & 1.366218 \\ \mathrm{C} & -0.572068 & -1.793407 & 1.341878 \\ \mathrm{C} & -1.634478 & -0.868925 & 1.358662 \\ \mathrm{C} & -1.732383 & 0.535477 & 1.402737 \\ \mathrm{C} & -0.807972 & 1.597363 & 1.452225 \\ \mathrm{C} & 0.597048 & 1.693990 & 1.483435\end{array}$




$\begin{array}{llll}\mathrm{H} & 0.944349 & 2.725152 & 1.363694 \\ \mathrm{H} & -1.287406 & 2.571350 & 1.311640 \\ \mathrm{H} & -2.756201 & 0.884816 & 1.235644 \\ \mathrm{H} & -2.601157 & -1.345530 & 1.167225 \\ \mathrm{H} & -0.913926 & -2.813874 & 1.141773 \\ \mathrm{H} & 1.318618 & -2.659695 & 1.179906 \\ \mathrm{H} & 2.787122 & -0.973339 & 1.270891 \\ \mathrm{H} & 2.631709 & 1.256536 & 1.349809 \\ \mathrm{C} & -0.249324 & 3.676441 & -3.207412 \\ \mathrm{H} & 0.305924 & 3.997000 & -2.310412 \\ \mathrm{H} & 0.490085 & 3.538431 & -4.013597 \\ \mathrm{H} & -0.911477 & 4.513111 & -3.484187 \\ \mathrm{C} & -2.533698 & 2.543142 & -3.289780 \\ \mathrm{H} & -2.784785 & 3.583989 & -3.551499 \\ \mathrm{H} & -2.824395 & 1.895204 & -4.132909 \\ \mathrm{H} & -3.175830 & 2.269177 & -2.436682 \\ \mathrm{H} & -0.943665 & 2.105225 & -1.894863\end{array}$

\section{$\left[\mathbf{T i}\left(\mathbf{N}^{t} \mathbf{B u}\right)(\mathbf{C O T})\left(\mathbf{C N}^{t} \mathbf{B u}\right)\right](\mathbf{1 4})$}

$\begin{array}{llll}\mathrm{C} & -0.505286 & 0.376241 & -0.623258 \\ \mathrm{Ti} & 3.168361 & -0.023812 & 0.014536 \\ \mathrm{C} & 2.248099 & 2.051759 & -0.304030 \\ \mathrm{C} & 2.648636 & 1.958366 & 1.039668 \\ \mathrm{C} & 3.745504 & 1.387530 & 1.713584 \\ \mathrm{C} & 4.897302 & 0.682250 & 1.317668 \\ \mathrm{C} & 5.425694 & 0.251373 & 0.086762 \\ \mathrm{C} & 5.021224 & 0.340235 & -1.259358 \\ \mathrm{C} & 3.919016 & 0.899627 & -1.933422 \\ \mathrm{C} & 2.770011 & 1.609832 & -1.537252 \\ \mathrm{~N} & -1.480028 & -0.247226 & -0.437511 \\ \mathrm{C} & -2.663515 & -1.006477 & -0.209948 \\ \mathrm{C} & -3.810443 & -0.315385 & -0.915665 \\ \mathrm{C} & -2.458087 & -2.403703 & -0.760504 \\ \mathrm{C} & -2.913295 & -1.056985 & 1.285436 \\ \mathrm{~N} & 2.444788 & -1.544705 & 0.179058 \\ \mathrm{C} & 1.728334 & -2.773388 & 0.255982 \\ \mathrm{C} & 0.668642 & -2.662609 & 1.341709 \\ \mathrm{C} & 1.073969 & -3.048470 & -1.088494 \\ \mathrm{C} & 2.688398 & -3.899879 & 0.596089 \\ \mathrm{H} & 0.483658 & -3.983054 & -1.058640 \\ \mathrm{H} & 0.412204 & -2.209316 & -1.362051 \\ \mathrm{H} & 1.848656 & -3.144852 & -1.867026 \\ \mathrm{H} & 2.152970 & -4.864554 & 0.658475 \\ \mathrm{H} & 3.468302 & -3.975812 & -0.179307 \\ \mathrm{H} & 3.177864 & -3.700655 & 1.563619 \\ \mathrm{H} & 0.005979 & -1.807985 & 1.123686\end{array}$




$\begin{array}{llll}\mathrm{H} & 0.067936 & -3.589113 & 1.406601 \\ \mathrm{H} & 1.147882 & -2.484780 & 2.318760 \\ \mathrm{H} & 1.862864 & 2.284408 & 1.729043 \\ \mathrm{H} & 1.208268 & 2.376373 & -0.400787 \\ \mathrm{H} & 2.043722 & 1.713465 & -2.350125 \\ \mathrm{H} & 3.883569 & 0.603746 & -2.986918 \\ \mathrm{H} & 5.633070 & -0.286436 & -1.916235 \\ \mathrm{H} & 6.271445 & -0.431460 & 0.217450 \\ \mathrm{H} & 5.435275 & 0.254663 & 2.169906 \\ \mathrm{H} & 3.608925 & 1.380457 & 2.799858 \\ \mathrm{H} & -3.373996 & -2.993361 & -0.591009 \\ \mathrm{H} & -2.251795 & -2.371849 & -1.842473 \\ \mathrm{H} & -1.612175 & -2.900745 & -0.259000 \\ \mathrm{H} & -3.828816 & -1.639098 & 1.479117 \\ \mathrm{H} & -2.069785 & -1.542787 & 1.802350 \\ \mathrm{H} & -3.045862 & -0.042189 & 1.692785 \\ \mathrm{H} & -4.736376 & -0.884068 & -0.742944 \\ \mathrm{H} & -3.947375 & 0.705112 & -0.529404 \\ \mathrm{H} & -3.627946 & -0.262536 & -1.998685\end{array}$

\section{$\left[\mathrm{Ti}\left(\mathrm{N}-2,{ }^{\mathrm{i}}-{ }^{\mathrm{i}} \mathrm{Pr}_{2} \mathrm{C}_{6} \mathrm{H}_{3}\right)(\mathrm{COT})\left(\mathrm{CN}^{\mathrm{t}} \mathrm{Bu}\right)\right]$ (17)}

$\begin{array}{llll}\mathrm{C} & 1.527782 & 7.204308 & 10.636456 \\ \mathrm{~N} & 0.836059 & 6.573506 & 9.568715 \\ \mathrm{C} & 0.259907 & 6.055473 & 8.691061 \\ \mathrm{Ti} & -0.843760 & 5.011294 & 7.167889 \\ \mathrm{~N} & -0.437072 & 6.220936 & 5.998289 \\ \mathrm{C} & -3.053574 & 4.793357 & 7.701391 \\ \mathrm{C} & -2.895115 & 4.173970 & 6.448674 \\ \mathrm{C} & -1.926543 & 3.349972 & 5.868222 \\ \mathrm{C} & -0.642188 & 2.921161 & 6.242028 \\ \mathrm{C} & 0.078128 & 2.902936 & 7.445801 \\ \mathrm{C} & -0.263918 & 3.213273 & 8.775215 \\ \mathrm{C} & -1.325153 & 3.872872 & 9.396066 \\ \mathrm{C} & -2.410004 & 4.639416 & 8.938577 \\ \mathrm{H} & -2.831030 & 5.283403 & 9.717836 \\ \mathrm{H} & -1.121528 & 4.056930 & 10.457266 \\ \mathrm{H} & 0.559039 & 3.010007 & 9.469269 \\ \mathrm{H} & 1.105416 & 2.543882 & 7.333238 \\ \mathrm{H} & -0.046242 & 2.594631 & 5.382890 \\ \mathrm{H} & -2.083404 & 3.249825 & 4.788187 \\ \mathrm{H} & -3.600559 & 4.561335 & 5.705248 \\ \mathrm{H} & -3.843193 & 5.550985 & 7.711922 \\ \mathrm{C} & 1.293998 & 8.699690 & 10.551913 \\ \mathrm{H} & 1.828463 & 9.195019 & 11.378932 \\ \mathrm{H} & 1.670357 & 9.098759 & 9.595908 \\ \mathrm{H} & 0.219968 & 8.932353 & 10.632865\end{array}$




$\begin{array}{llll}\mathrm{C} & 0.982611 & 6.650313 & 11.938894 \\ \mathrm{H} & 1.515083 & 7.117087 & 12.783434 \\ \mathrm{H} & -0.092607 & 6.870126 & 12.035196 \\ \mathrm{H} & 1.130187 & 5.559561 & 11.989483 \\ \mathrm{C} & 3.005248 & 6.885404 & 10.501060 \\ \mathrm{H} & 3.556357 & 7.364536 & 11.326001 \\ \mathrm{H} & 3.174479 & 5.797936 & 10.545457 \\ \mathrm{H} & 3.400406 & 7.265031 & 9.544966 \\ \mathrm{C} & -0.219352 & 7.188850 & 5.068784 \\ \mathrm{C} & -0.446750 & 6.896724 & 3.701130 \\ \mathrm{C} & -0.254037 & 7.869820 & 2.761424 \\ \mathrm{C} & 0.132114 & 9.132743 & 3.091611 \\ \mathrm{C} & 0.355735 & 9.444884 & 4.422827 \\ \mathrm{C} & 0.201358 & 8.494478 & 5.418550 \\ \mathrm{C} & 0.581789 & 8.794695 & 6.836511 \\ \mathrm{C} & 0.353804 & 10.237373 & 7.212157 \\ \mathrm{H} & 0.517355 & 10.398268 & 8.292007 \\ \mathrm{H} & 1.062790 & 10.898518 & 6.684280 \\ \mathrm{H} & -0.667397 & 10.569667 & 6.963998 \\ \mathrm{C} & 2.039454 & 8.385216 & 7.043788 \\ \mathrm{H} & 2.695039 & 9.273491 & 7.004590 \\ \mathrm{H} & 2.203852 & 7.880454 & 8.008697 \\ \mathrm{H} & 2.374705 & 7.694602 & 6.254014 \\ \mathrm{H} & -0.058425 & 8.139530 & 7.458121 \\ \mathrm{H} & 0.666421 & 10.458501 & 4.699426 \\ \mathrm{H} & 0.268628 & 9.893413 & 2.321683 \\ \mathrm{H} & -0.425153 & 7.613354 & 1.709492 \\ \mathrm{C} & -0.870731 & 5.515090 & 3.289909 \\ \mathrm{C} & -2.077059 & 5.540519 & 2.349821 \\ \mathrm{H} & -2.282084 & 6.557090 & 1.983266 \\ \mathrm{H} & -1.914548 & 4.913010 & 1.461042 \\ \mathrm{H} & -2.990943 & 5.189767 & 2.850552 \\ \mathrm{C} & 0.303113 & 4.746524 & 2.683812 \\ \mathrm{H} & 0.843461 & 5.377501 & 1.960618 \\ \mathrm{H} & 1.006667 & 4.407872 & 3.460735 \\ \mathrm{H} & -0.056828 & 3.857414 & 2.141609 \\ \mathrm{H} & -1.142687 & 5.005039 & 4.225815\end{array}$

\section{[Ti(N'Bu)(COT)(py)] (17)}

$\begin{array}{llll}\mathrm{Ti} & 2.300160 & 4.083285 & 6.961892 \\ \mathrm{~N} & 2.751506 & 5.165437 & 5.724164 \\ \mathrm{C} & 3.075083 & 6.208589 & 4.815718 \\ \mathrm{C} & 4.383665 & 6.872088 & 5.216025 \\ \mathrm{C} & 1.954866 & 7.238211 & 4.817530 \\ \mathrm{C} & 3.213712 & 5.613684 & 3.423180 \\ \mathrm{~N} & 3.445765 & 5.426884 & 8.297856\end{array}$




$\begin{array}{llll}\mathrm{C} & 4.616586 & 5.109236 & 8.855016 \\ \mathrm{C} & 5.327324 & 5.986968 & 9.655135 \\ \mathrm{C} & 4.796276 & 7.238680 & 9.911737 \\ \mathrm{C} & 3.581621 & 7.572659 & 9.332386 \\ \mathrm{C} & 2.951177 & 6.648032 & 8.522373 \\ \mathrm{C} & 1.855776 & 3.173130 & 9.289364 \\ \mathrm{C} & 0.769276 & 3.916853 & 8.810088 \\ \mathrm{C} & 0.099846 & 4.009897 & 7.578287 \\ \mathrm{C} & 0.210344 & 3.318922 & 6.357978 \\ \mathrm{C} & 1.124711 & 2.408319 & 5.814207 \\ \mathrm{C} & 2.378172 & 1.918048 & 6.207285 \\ \mathrm{C} & 3.132611 & 1.955986 & 7.394157 \\ \mathrm{C} & 2.861919 & 2.405234 & 8.697494 \\ \mathrm{H} & 4.638130 & 7.687937 & 4.516012 \\ \mathrm{H} & 4.307968 & 7.297067 & 6.232789 \\ \mathrm{H} & 5.201070 & 6.131879 & 5.209459 \\ \mathrm{H} & 2.145665 & 8.038635 & 4.080441 \\ \mathrm{H} & 1.864163 & 7.697948 & 5.817034 \\ \mathrm{H} & 0.997900 & 6.747864 & 4.573776 \\ \mathrm{H} & 3.445501 & 6.399184 & 2.681432 \\ \mathrm{H} & 4.021608 & 4.863776 & 3.415230 \\ \mathrm{H} & 2.274461 & 5.113928 & 3.133713 \\ \mathrm{H} & 4.987824 & 4.100282 & 8.643640 \\ \mathrm{H} & 6.288697 & 5.679026 & 10.075799 \\ \mathrm{H} & 5.325239 & 7.950444 & 10.553737 \\ \mathrm{H} & 3.119263 & 8.548964 & 9.502309 \\ \mathrm{H} & 2.003286 & 6.876235 & 8.021009 \\ \mathrm{H} & 2.106301 & 3.444602 & 10.320766 \\ \mathrm{H} & 0.391008 & 4.633067 & 9.548074 \\ \mathrm{H} & -0.667779 & 4.790685 & 7.559782 \\ \mathrm{H} & -0.482298 & 3.701888 & 5.601670 \\ \mathrm{H} & 0.958402 & 2.269364 & 4.741652 \\ \mathrm{H} & 2.938372 & 1.495080 & 5.367654 \\ \mathrm{H} & 4.126769 & 1.510356 & 7.285598 \\ \mathrm{H} & 3.692950 & 2.213053 & 9.385625\end{array}$

$\begin{array}{lccc}\mathbf{T i}(\mathbf{C O T})\left(\eta^{\mathbf{4}}-\mathbf{C}_{\mathbf{8}} \mathbf{H}_{\mathbf{8}}\right) & \mathbf{( 1 3}) & \\ \mathrm{Ti} & 8.176724 & 15.906082 & 3.138393 \\ \mathrm{C} & 6.680300 & 17.338407 & 2.552312 \\ \mathrm{C} & 6.158553 & 16.485810 & 3.556645 \\ \mathrm{C} & 6.533200 & 16.341319 & 4.909580 \\ \mathrm{C} & 7.031966 & 17.196411 & 5.915003 \\ \mathrm{C} & 7.539686 & 18.479399 & 5.953610 \\ \mathrm{C} & 8.028874 & 19.336467 & 4.962533 \\ \mathrm{C} & 8.189968 & 19.195268 & 3.598844 \\ \mathrm{C} & 7.701709 & 18.307935 & 2.618327\end{array}$




$\begin{array}{llll}\mathrm{H} & 8.073297 & 18.557851 & 1.615428 \\ \mathrm{H} & 8.843088 & 19.962244 & 3.161600 \\ \mathrm{H} & 8.468803 & 20.261203 & 5.360068 \\ \mathrm{H} & 7.680469 & 18.861751 & 6.972882 \\ \mathrm{H} & 6.980564 & 16.725724 & 6.906935 \\ \mathrm{H} & 6.170549 & 15.389346 & 5.320581 \\ \mathrm{H} & 5.455797 & 15.728494 & 3.184708 \\ \mathrm{H} & 6.316112 & 17.115575 & 1.540301 \\ \mathrm{C} & 8.745889 & 14.658482 & 1.324585 \\ \mathrm{C} & 8.238901 & 13.792458 & 2.314061 \\ \mathrm{C} & 8.394360 & 13.668751 & 13.705261 \\ \mathrm{C} & 9.058555 & 14.409525 & 4.690578 \\ \mathrm{C} & 9.837499 & 15.576131 & 4.693685 \\ \mathrm{C} & 10.334776 & 16.441776 & 3.710823 \\ \mathrm{C} & 10.268257 & 16.502728 & 2.310704 \\ \mathrm{C} & 9.613946 & 15.762368 & 1.319021 \\ \mathrm{H} & 9.647071 & 16.254950 & 0.341430 \\ \mathrm{H} & 10.687127 & 17.436013 & 1.920481 \\ \mathrm{H} & 10.763536 & 17.352606 & 4.144674 \\ \mathrm{H} & 9.977524 & 15.978175 & 5.703462 \\ \mathrm{H} & 8.766877 & 14.107276 & 5.701526 \\ \mathrm{H} & 7.710473 & 12.927254 & 4.131170 \\ \mathrm{H} & 7.457934 & 13.132176 & 1.922513 \\ \mathrm{H} & 8.261778 & 14.504115 & 0.354481\end{array}$

\begin{tabular}{llll}
\multicolumn{2}{l}{$\mathbf{B u N C}$} & & \\
$\mathrm{C}$ & 4.984745 & 7.032911 & 10.142382 \\
$\mathrm{~N}$ & 4.226866 & 6.225323 & 9.252662 \\
$\mathrm{C}$ & 3.594124 & 5.565523 & 8.519837 \\
$\mathrm{C}$ & 4.699803 & 8.489002 & 9.833549 \\
$\mathrm{H}$ & 5.272694 & 9.123022 & 10.529646 \\
$\mathrm{H}$ & 4.996127 & 8.737354 & 8.802242 \\
$\mathrm{H}$ & 3.627833 & 8.707567 & 9.957662 \\
$\mathrm{C}$ & 4.566462 & 6.703926 & 11.562097 \\
$\mathrm{H}$ & 5.144866 & 7.327111 & 12.263134 \\
$\mathrm{H}$ & 3.494292 & 6.908728 & 11.706977 \\
$\mathrm{H}$ & 4.758198 & 5.643463 & 11.787169 \\
$\mathrm{C}$ & 6.455728 & 6.723230 & 9.937823 \\
$\mathrm{H}$ & 7.058918 & 7.389104 & 10.576154 \\
$\mathrm{H}$ & 6.674531 & 5.677964 & 10.208559 \\
$\mathrm{H}$ & 6.739695 & 6.884172 & 8.885862
\end{tabular}

\begin{tabular}{llll}
\multicolumn{4}{c}{ 1,3,5,7-Cyclooctatetraene } \\
C & 0.614743 & -1.560407 & -0.389055 \\
C & 1.560407 & -0.614743 & -0.389055 \\
C & 1.560407 & 0.614743 & 0.389055
\end{tabular}




$\begin{array}{llll}\mathrm{C} & 0.614743 & 1.560407 & 0.389055 \\ \mathrm{C} & -0.614743 & 1.560407 & -0.389055 \\ \mathrm{C} & -1.560407 & 0.614743 & -0.389055 \\ \mathrm{C} & -1.560407 & -0.614743 & 0.389055 \\ \mathrm{C} & -0.614743 & -1.560407 & 0.389055 \\ \mathrm{H} & -0.815068 & -2.478122 & 0.961304 \\ \mathrm{H} & -2.478122 & -0.815068 & 0.961304 \\ \mathrm{H} & -2.478122 & 0.815068 & -0.961304 \\ \mathrm{H} & -0.815068 & 2.478122 & -0.961304 \\ \mathrm{H} & 0.815068 & 2.478122 & 0.961304 \\ \mathrm{H} & 2.478122 & 0.815068 & 0.961304 \\ \mathrm{H} & 2.478122 & -0.815068 & -0.961304 \\ \mathrm{H} & 0.815068 & -2.478122 & -0.961304\end{array}$

\section{${ }^{t} B^{\prime} N_{C N}{ }^{t} B u$}

$\begin{array}{llll}\mathrm{N} & 1.346271 & 1.131034 & -0.938656 \\ \mathrm{C} & 1.975132 & 2.264811 & -1.590637 \\ \mathrm{C} & 2.360410 & 3.233860 & -0.492401 \\ \mathrm{H} & 2.810175 & 4.144040 & -0.922308 \\ \mathrm{H} & 1.468927 & 3.515437 & 0.089947 \\ \mathrm{H} & 3.086627 & 2.767563 & 0.192687 \\ \mathrm{C} & 3.198770 & 1.846996 & -2.372912 \\ \mathrm{H} & 3.694153 & 2.727710 & -2.814678 \\ \mathrm{H} & 3.921218 & 1.334661 & -1.715678 \\ \mathrm{H} & 2.924600 & 1.158437 & -3.191264 \\ \mathrm{C} & 0.957833 & 2.911549 & -2.505550 \\ \mathrm{H} & 1.375087 & 3.834335 & -2.942985 \\ \mathrm{H} & 0.680701 & 2.228537 & -3.325477 \\ \mathrm{H} & 0.046643 & 3.167793 & -1.941736 \\ \mathrm{C} & 1.598855 & -0.058580 & -1.041636 \\ \mathrm{~N} & 1.851585 & -1.248779 & -0.946903 \\ \mathrm{C} & 1.221639 & -2.378053 & -1.605438 \\ \mathrm{C} & 0.852513 & -3.362636 & -0.515305 \\ \mathrm{H} & 0.131364 & -2.908333 & 0.183204 \\ \mathrm{H} & 0.401963 & -4.268988 & -0.952437 \\ \mathrm{H} & 1.751443 & -3.647662 & 0.053826 \\ \mathrm{C} & -0.012511 & -1.957153 & -2.369308 \\ \mathrm{H} & 0.249116 & -1.258581 & -3.183120 \\ \mathrm{H} & -0.510016 & -2.834813 & -2.814367 \\ \mathrm{H} & -0.728911 & -1.454655 & -1.698399 \\ \mathrm{C} & 2.233185 & -3.007940 & -2.538694 \\ \mathrm{H} & 2.498440 & -2.312877 & -3.352576 \\ \mathrm{H} & 3.151106 & -3.265934 & -1.986576 \\ \mathrm{H} & 1.816958 & -3.927784 & -2.983609\end{array}$

${ }^{\mathrm{t}} \mathrm{BuNCN}-\left(2,6-{ }^{\mathrm{i}} \mathrm{Pr}_{2} \mathrm{C}_{6} \mathrm{H}_{3}\right)$ 


\begin{tabular}{|c|c|c|c|}
\hline $\mathrm{N}$ & 1.427580 & 0.937869 & -0.770129 \\
\hline $\mathrm{C}$ & 1.904823 & 2.144017 & -1.429116 \\
\hline $\mathrm{C}$ & 2.216876 & 3.155064 & -0.349535 \\
\hline $\mathrm{H}$ & 2.569858 & 4.096718 & -0.800898 \\
\hline $\mathrm{H}$ & 1.316439 & 3.363792 & 0.249458 \\
\hline $\mathrm{H}$ & 2.999591 & 2.770282 & 0.323583 \\
\hline $\mathrm{C}$ & 3.145549 & 1.839858 & -2.237791 \\
\hline $\mathrm{H}$ & 3.524581 & 2.755223 & -2.721930 \\
\hline $\mathrm{H}$ & 3.939680 & 1.434963 & -1.588703 \\
\hline $\mathrm{H}$ & 2.924812 & 1.097596 & -3.024799 \\
\hline $\mathrm{C}$ & 0.804992 & 2.669999 & -2.326576 \\
\hline $\mathrm{H}$ & 1.121778 & 3.616285 & -2.797108 \\
\hline $\mathrm{H}$ & 0.572251 & 1.943637 & -3.122672 \\
\hline $\mathrm{H}$ & -0.110487 & 2.857314 & -1.743214 \\
\hline $\mathrm{C}$ & 1.541046 & -0.223272 & -1.116245 \\
\hline $\mathrm{N}$ & 1.745400 & -1.410260 & -1.300677 \\
\hline $\mathrm{C}$ & 1.060745 & -2.479406 & -1.855012 \\
\hline $\mathrm{C}$ & -0.286048 & -2.716630 & -1.542955 \\
\hline $\mathrm{C}$ & -0.895631 & -3.831601 & -2.102692 \\
\hline $\mathrm{C}$ & -0.200996 & -4.685881 & -2.943030 \\
\hline $\mathrm{C}$ & 1.127928 & -4.433217 & -3.236651 \\
\hline $\mathrm{C}$ & 1.781974 & -3.337431 & -2.692622 \\
\hline $\mathrm{C}$ & 3.236195 & -3.064425 & -2.939408 \\
\hline $\mathrm{C}$ & 4.052864 & -3.573500 & -1.764881 \\
\hline $\mathrm{H}$ & 3.691318 & -3.143073 & -0.818142 \\
\hline $\mathrm{H}$ & 3.968413 & -4.672695 & -1.700283 \\
\hline $\mathrm{H}$ & 5.120102 & -3.319884 & -1.883956 \\
\hline $\mathrm{C}$ & 3.748466 & -3.641895 & -4.235977 \\
\hline $\mathrm{H}$ & 3.174133 & -3.277072 & -5.103233 \\
\hline $\mathrm{H}$ & 4.806837 & -3.370140 & -4.382089 \\
\hline $\mathrm{H}$ & 3.694761 & -4.744625 & -4.232451 \\
\hline $\mathrm{H}$ & 3.352959 & -1.965064 & -2.971725 \\
\hline $\mathrm{H}$ & 1.675773 & -5.101224 & -3.909502 \\
\hline $\mathrm{H}$ & -0.703347 & -5.555306 & -3.376386 \\
\hline $\mathrm{H}$ & -1.948215 & -4.028538 & -1.876275 \\
\hline $\mathrm{C}$ & -1.010437 & -1.802703 & -0.598867 \\
\hline $\mathrm{C}$ & -2.508626 & -1.989490 & -0.618331 \\
\hline $\mathrm{H}$ & -2.993199 & -1.235773 & 0.022363 \\
\hline $\mathrm{H}$ & -2.926436 & -1.903658 & -1.634240 \\
\hline $\mathrm{H}$ & -2.784332 & -2.981272 & -0.218758 \\
\hline $\mathrm{C}$ & -0.487020 & -1.972236 & 0.817613 \\
\hline $\mathrm{H}$ & -0.966961 & -1.249429 & 1.498427 \\
\hline $\mathrm{H}$ & -0.714501 & -2.990323 & 1.179753 \\
\hline $\mathrm{H}$ & 0.603486 & -1.826546 & 0.872024 \\
\hline $\mathrm{H}$ & -0.793092 & -0.761746 & -0.911857 \\
\hline
\end{tabular}




\section{References}

1. Thorman, J. L.; Guzei, I. A.; Young Jr., V. G.; Woo, K. L. Inorg. Chem. 1999, 38, 3814-3824.

2. Guiducci, A. E.; Boyd, C. L.; Mountford, P. Organometallics accepted subject to minor revisions (OM050784V). 